\title{
Surface-enhanced Raman spectroscopy for identification and discrimination of beverage spoilage yeasts using patterned substrates and gold nanoparticles
}

\author{
Sanna Uusitalo, ${ }^{a}$ Alexey Popov, ${ }^{b}, y, z$ Yury V. Ryabchikov, ${ }^{c, d}$ Olga Bibikova, ${ }^{b}$ Hanna-Leena \\ Alakomi, ${ }^{a}$ Riikka Juvonen, ${ }^{a}$ Ville Kontturi, ${ }^{e}$ Samuli Siitonen, ${ }^{e}$ Andrei Kabashin, ${ }^{c}$ Igor \\ Meglinski, ${ }^{b, x, y, z}$ Jussi Hiltunen ${ }^{a^{*}}$, Arja Laitila $^{a^{*}}$

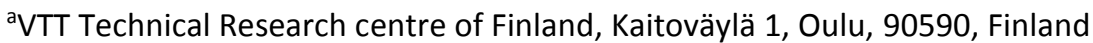 \\ b Optoelectronics and Measurement Techniques, Faculty of Information Technology and Electrical Engineering, \\ University of Oulu, Erkki-Koiso Kanttilan katu 3, Oulu, 90570 Finland \\ 'Aix-Marseille University, CNRS, UMR 7341 CNRS, LP3, Campus de Luminy, Case 917, F-13288, Marseille Cedex 9, \\ France \\ dP.N. Lebedev Physical Institute of Russian Academy of Sciences, 53 Leninskii Prospekt, Moscow 199 991, Russia \\ eNanocomp Oy Ltd, Ensolantie 6, Lehmo, Finland, 80710 \\ fNational Research Nuclear University “MEPhI”, Institute of Engineering Physics for Biomedicine (PhysBio), 115409 \\ Moscow, Russia \\ z Terahertz Biomedicine Laboratory, ITMO University, 49 Kronverksky Prospekt, St. Petersburg, 197101, Russia \\ × Laboratory of Biosensorics and Eco-Photonics, Irkutsk State University, Irkutsk, 664003, Russia \\ ${ }^{y}$ Interdisciplinary Laboratory of Biophotonics, Tomsk National Research State University, 634050 Tomsk, Russia
}

Corresponding Author Sanna Uusitalo (sanna.uusitalo@vtt.fi)

* Equally shared supervision

Keywords: beverage, yeast, Raman spectroscopy, SERS, nanoparticles

\begin{abstract}
In the beverage industry, the detection of spoilage yeasts such as Wickerhamomyces anomalus and Brettanomyces bruxellensis can be labourious and time-consuming. In the present study, a simple and repeatable technique was developed for rapid yeast detection using a combination of patterned goldcoated surface-enhanced Raman spectroscopy (SERS) substrates and gold nanoparticles. W. anomalus and B. bruxellensis showed several characteristic peaks, enabling the discrimination of these yeasts without chemometric analysis. The control yeast used as an indicator yeast, Rhodotorula mucilaginosa, showed 7 cell wall-related peaks originating from lipids and haemoproteins. AnalysingW. anomalus SERS spectra with differently sized and shaped gold nanoparticles revealed the benefit of using either large, spherical, chemically synthesised gold nanoparticles or small, laser-synthesised, gold-silicon nanoparticles for yeast detection. Additionally, the spectra showed differences in SERS signal construction for small molecules and biological cells, as the nanoparticles with best response in biological cell detection did not excel in small molecule detection. The use of small composite gold-silicon nanoparticles in combination with the SERS substrate gave distinctive spectra for all detected yeast species.
\end{abstract}

\section{Introduction}

Yeast spoilage in the beverage industry is a costly problem arising from off-flavors caused by the metabolic end products of yeast cells $\mathrm{s}^{1,2}$. Although yeasts are an essential part in making beer and wine and 
are often vital in forming the specific taste of the beverage ${ }^{3,4}$, some yeasts can pose a problem, since they can spoil the final products. In worst case the yeast spoilage can include exploded cans of soft drinks, cloudy re-fermented products with unwanted off-flavour in beverages ${ }^{3,5}$. The presence of a few cells of specific spoilage yeast at any stage of the manufacturing process may result in quality defects perceptible to consumer. Despite of many advances in controlling microbiological risks in beverage production, the globalization of trade, the use of exotic raw materials, novel processing technologies and the growing demand of minimally processed fresh-like products create new challenges for manufacturers to ensure the product safety and stability. Thus, there is a need to develop effective control and detection tools for harmful microbes including spoilage yeasts.

Beverages containing spoilage yeasts are difficult to detect by naked eye as it takes up to 10000 cells $/ \mathrm{ml}$ to make a difference in the appearance of the drink. In the beverage industry, microbiological quality control mainly relies on the use of plate cultivation techniques, often laborious and time-consuming (typically 5-7 days). Furthermore, they rarely distinguish between spoilage and harmless microbes. After detection the identification of the yeasts often requires slow and laborious DNA sequencing or morphological and physiological testing ${ }^{1,2,6,7}$.

Recently, surface-enhanced Raman spectroscopy (SERS) for the identification of yeasts has been demonstrated by several research groups. ${ }^{8-13}$ SERS detection is based on vibrational spectroscopy where the signal intensity of Raman scattering is enhanced by localized or propagating plasmon resonance in noble metals. ${ }^{14}$ Thus, the bonds of the biomolecules, such as proteins, lipids, carbohydrates, and nucleic acids, on the cell wall of yeast cells, create a specific Raman spectrum enabling identification of different yeast species. SERS is promising for molecular and microbial detection with such advantages as analyte identification, speed and possibility for multiple simultaneous analyses. The presence of other microbes or DNA in the laboratory is not a concern in SERS. ${ }^{5}$ The yeast detection by SERS has been mainly focused on characterization of different yeasts with colloidal suspensions of silver nanoparticles (AgNPs) incubated with the cells, and by focussing on single cells or small clusters of cells in combination with highly magnifying optics. ${ }^{8-10,12,15,16}$ 
Syamala et al. revealed the difficulty in obtaining reproducible SERS spectra from the yeast cells with AgNPs incubation as the proteins in the cell walls have various factors affecting the adsorption of AgNPs including the flexibility/mobility, accessibility, polarity and the exposed surface. ${ }^{12}$ AgNPs are advantageous for SERS as silver has a higher SERS enhancement factor than other metals such as gold. ${ }^{17}$ However, there are reasons for considering the latter for SERS. AgNPs are prone to oxidation and release of toxic silver ions, and although surface stabilization is applied, they can be bio-incompatible and inappropriate for longer storage ${ }^{18}$. Gold on the other hand is stable and non-toxic, and does not harm the yeast cells under inspection ${ }^{19,20}$. Additionally, gold-initiated SERS can be excited with near-infrared (NIR) wavelengths, thus reducing thermal damage due to longer wavelengths with lower energy. ${ }^{21}$

Up to now, only very little research has been performed on the Raman or the SERS detection of other beverage yeasts than the Saccharomyces cerevisiae (S. cerevisiae). Rodriguez et al. measured the Raman spectra of wine spoilage yeasts including S. cerevisiae, Zygosaccharomyces bailii (Z. bailii), and Brettanomyces bruxellensis (B. bruxellensis), but specific Raman spectrum with several peaks for the B. bruxellensis or the other yeasts of the study were not observed. The study was executed with microscope-assisted Raman without SERS enhancement and relatively high excitation laser power ( $30 \mathrm{~mW})$ was used..$^{5}$ In the present study, the yeast detection by SERS was developed by NIR excitation, reduced laser power and thermally dried yeast cells on top of SERS substrate with AuNPs to achieve characteristic Raman spectra for straight and simple discrimination of the beverage yeast species. Incubation of AuNPs with yeast cells prior to detection was avoided to obtain spectra with less variance due to differing colloid adsorption factors. Raman spectra for two beverage spoilage yeasts, Wickerhamomyces anomalus (W. anomalus) and B. bruxellensis, and a reference yeast, $R$. mucilaginosa were successfully detected. Different AuNPs for the detection of $W$. anomalus were analysed to find the optimal combination of SERS substrate and NPs producing high SERS enhancement with low background signal. The SERS substrates were used as an analysis chip basis and to increase electromagnetic field with the combination of NPs 22,23 . They were fabricated with roll-to-roll UVnanoimprint lithography allowing for high-throughput manufacturing of the SERS chips $^{24}$. 


\section{Materials and Methods}

\section{Yeast cultures:}

Yeast strains isolated from beverage production were obtained from VTT Culture Collection (http://culturecollection.vtt.fi): W. anomalus VTT C-02470 (C470), B. bruxellensis VTT C-05796 (C796) and R. mucilaginosa VTT C-89179 (C179). Yeast strains were grown on yeast-malt extract agar, YM-agar (Difco Laboratories, USA) at $25^{\circ} \mathrm{C}$ for 2-4 days. Yeast samples in $0.9 \%$ physiological saline were prepared by using a precision scale to measure the ratio of loop ('loop' - what does this mean here?) inserted yeast mass and the saline. W. anomalus concentration for the nanoparticle analysis was $8 \cdot 10^{7}$ cells $/ \mathrm{ml}$, prepared by adding $0.015 \mathrm{~g}$ of yeast to $2 \mathrm{ml}$ of saline. W. anomalus, B. bruxellensis and R. mucilaginosa concentrations for gold-silicon composite nanoparticle (AuSi NC) detection was $4 \cdot 10^{7}$ cells $/ \mathrm{ml}$.

\section{Gold nanoparticles:}

Star nanoparticles:

Gold nanostars (Star AuNPs) were fabricated by seed-mediated growth method $^{25}$ with slight modifications. ${ }^{26,27} 15-\mathrm{nm}$ gold nanospheres (NSps) were used as seeds. In detail, $10 \mu \mathrm{L}$ of $1 \mathrm{M} \mathrm{HCl}$ and $100 \mu \mathrm{L}$ of the NSps solution were added to $10 \mathrm{~mL}$ of $0.25 \mathrm{mM} \mathrm{HAuCl}_{4}$ water solution at room temperature under vigorous stirring. $100 \mu \mathrm{L}$ of $2 \mathrm{mM} \mathrm{AgNO}_{3}$ and $50 \mu \mathrm{L}$ of $0.1 \mathrm{M}$ ascorbic acid were added to the solution with 30 s stirring as the colour changed to dark grey. $250 \mu \mathrm{L}$ of $0.36 \mathrm{mM}$ polyvinyl pyrrolidone (PVP) was added under gentle stirring for $15 \mathrm{~min}$. The solution was centrifuged twice at a 7500 relative centrifugal force in terms of acceleration for 10 min. $15-\mathrm{nm}$ star AuNPs were synthesized by the reduction of $\mathrm{HAuCl}_{4}$ with sodium citrate by Grabar's method: $4.5 \mathrm{ml}$ of $1 \%$ sodium citrate was added quickly to boiling $0.03 \% \mathrm{HAuCl}_{4}(50 \mathrm{ml})$ resulting in a change in solution colour to deep orange-red. The original star AuNP concentration was diluted with a ratio of 1:4 in deionised (DI) water to make it closer to OD of spherical particles.

Ultrapure nanoparticles: 
Synthesis of gold-silicon nanocomposite particles (AuSi NCs) was carried out by means of the pulsed laser ablation method which has been described previously. ${ }^{28-32}$ In brief, a gold target was ablated in a solution of previously prepared silicon NPs (SiNPs). Laser ablation was performed by Ti:Sapphire laser (Hurricane Spectra Physics Laser, USA) operated at 800-nm wavelength and $1000 \mathrm{~Hz}$ repetition rate with $110 \mathrm{fs}$ pulse duration. Laser energy fluence was fixed at $150 \mu \mathrm{J} /$ pulse. For fabrication of colloidal solution of SiNPs ${ }^{28,29}$, a Si target was immersed in $18 \mathrm{M} \Omega$ deionized water and exposed to fs pulsed laser radiation. The Si target was replaced by an Au target and laser ablation procedure was applied in the presence of SiNPs. ${ }^{31}$ The dual ablation leads to the formation of AuSi composite NPs (AuSi NC). ${ }^{32}$ The advantage of laser-assisted synthesis is in the absence of ligands on the NP surface, which guarantees a strong response of formed NPs. ${ }^{33,34}$ Despite the presence of protective ligands, AuSi NCs are extremely stable due to strong negative charge of colloids leading to electric repulsion effect. The original AuSi NC suspension was diluted with a ratio of 1:1 in DI water to make it closer to OD of spherical particles.

Chemically synthesized spherical nanoparticles:

Spherical NPs of different sizes were purchases (Sigma-Aldrich, CytoDiagnostics, USA). NPs $10 \mathrm{~nm}, 80 \mathrm{~nm}$, $150 \mathrm{~nm}$ and $200 \mathrm{~nm}$ in diameter were acquired in $0.1 \mathrm{mg} / \mathrm{ml}$ citrate buffer. According to the description, the NPs in citrate buffer have a high salt stability $(30 \mathrm{mM} \mathrm{NaCl}, 30 \mathrm{~min})$. OD of NPs was set to 1 . The concentrations of $200-\mathrm{nm}, 150-\mathrm{nm}, 80-\mathrm{nm}$ and $10-\mathrm{nm}$ NPs in citrate buffer were $1.9 \cdot 10^{9} \mathrm{particles} / \mathrm{ml}, 3.6 \cdot 10^{9} \mathrm{particles} / \mathrm{ml}$, 7.8.10 $10^{9}$ particles $/ \mathrm{mL}, 6.0 \cdot 10^{12}$ particles $/ \mathrm{ml}$, respectively. $150-\mathrm{nm}$ NPs were also obtained in phosphate buffered saline (PBS) buffer. The NP concentration was 3.6.10 particles $/ \mathrm{mL}$, OD was 1 and the solution was $99 \%$ free of residual reactants from manufacturing.

\section{SERS substrate and PDMS sample well:}

SERS substrate fabrication and PDMS well integration has been described by S. Uusitalo et ll. $^{23}$ and S.Z. Oo et al. ${ }^{24}$. In short, the SERS substrates were fabricated by using UV-nanoimprint lithography and roll-to-roll (R2R) equipment. UV-curable lacquer was applied with reverse gravure and were patterned with embossing reel and the structures were cured through UV exposure. The web was die-cut and coated with a 240-nm 
gold layer by vapour deposition. 2-mm hydrophobic sample wells were integrated to the gold layer of the SERS substrates by physical adsorption. The developed R2R fabrication is capable of high throughput volumes with low chip cost simplifying the detection as the chips can be disposed after use and do not require extra cleaning steps.

\section{UV-VIS spectroscopy and TEM imaging:}

UV-Vis spectroscopy was performed using a spectrophotometer (Optronic Laboratories, USA) within 3501050-nm wavelength range. Originally measured collimated transmittance was recalculated into OD. OD of different NPs was tuned into similar range by diluting the AuSi NCs from the original concentration to 1:1 ratio and the star AuNPs to 1:4 ratio. The diluted samples were used in the SERS detection of $W$. anomalus.

TEM imaging was performed with LEO 912 Omega EFTEM (Zeiss, Germany) by drop-casting NP suspensions onto carbon-coated copper grids.

\section{Raman spectroscopy:}

785-nm excitation laser was used in a Raman system coupled with an optical microscope (Olympus, Japan) in combination with 50x magnifying optics. Spectra were acquired over a Raman shift range of 400 to $2100 \mathrm{~cm}^{-1}$ with a $1 \mathrm{~cm}^{-1}$ resolution. $100 \mu \mathrm{M}$ Rhodamine $6 \mathrm{G}$ (R6G) was mixed with $0.1 \%$ non-ionic detergent TWEEN in volume ratio of $70: 30$ and dried on top of the hot plate $\left(40^{\circ} \mathrm{C}\right)$ inside $1.5-\mathrm{mm}$ diameter PDMS well integrated on the SERS substrate. Subsequently AuNP solutions were dried on top of the R6G samples (40 $\left.{ }^{\circ} \mathrm{C}\right)$. Integration time used with duplicate samples: diluted star AuNPs, AuSi NCs and 150-nm AuNPs in PBS $1 \mathrm{~s}$, rest of the particles $2 \mathrm{~s} .15$ points were recorded per sample and results were normalised by the integration time. Yeast response results were averaged from three collected spectra, with 3 times averaged $5 \mathrm{~s}$ integration times each. Measurements of each yeast specie were done in triplicate with PDMS wells 2$\mathrm{mm}$ in diameter, and 10 measurement points with about $30-\mu \mathrm{m}$ spacing were recorded per one well (30 measurement points/sample). The yeast samples with $4-\mu l$ volume were pipetted inside the wells integrated onto the SERS substrates and dried at $30^{\circ} \mathrm{C}$. The drying caused the salts of the buffer to crystallise locally. 
Raman detection was conducted over areas free of the crystallised salt. 4- $\mu$ I AuNP solutions were dried on top of the yeast samples at $30^{\circ} \mathrm{C}$.

\section{Results and discussion}

Two spoilage yeasts, W. anomalus and B. bruxellensis, and additional control yeast, R. mucilaginosa, were chosen for this study to develop a new method for the beverage yeast detection by SERS. W. anomalus and B. bruxellensis are often related to the production of volatile compounds causing off-flavor in wines, beers and soft drinks ${ }^{35}$. B.rettanomyces bruxellensis, also known as Dekkera bruxellensis, can develop an acetic taste in beers and undesirable flavours in soft drinks due to acetate-ester production. ${ }^{36}$ R. mucilaginosa is a soil yeast, which produces red/pink colored carotenoids ${ }^{37}$. In the beverage industry, this type of yeast is often considered as hygiene indicators at the manufacturing plants, but they are not normally associated with spoilage of the final product. ${ }^{38}$

The suitability of star AuNPs, gold nanospheres of different sizes and bare AuSi NCs were studied for SERS detection of yeast strains. The effect of size, shape and buffer of NPs on Raman spectra was analysed with light spectroscopy, TEM imaging and SERS detection using model analyte Rhodamine 6G (R6G) and the chosen yeast strains. These parameters can affect the NPs plasmon resonance wavelength and thus the SERS enhancement capability. ${ }^{39}$ TEM imaging was used to compare the shapes and reveal NP size distribution. All NPs had spherical shapes as shown in Figure 1 a) -g). The star AuNPs shown in Figure 1 a) have additional sharp spikes affecting their VIS-NIR absorbance and creating SERS hotspots. The size ranges of the commercial particles and star AuNPs are consistent but the AuSi NCs as shown in Figure $1 \mathrm{~b}$ ) have few larger particles in the midst of the small ones. 


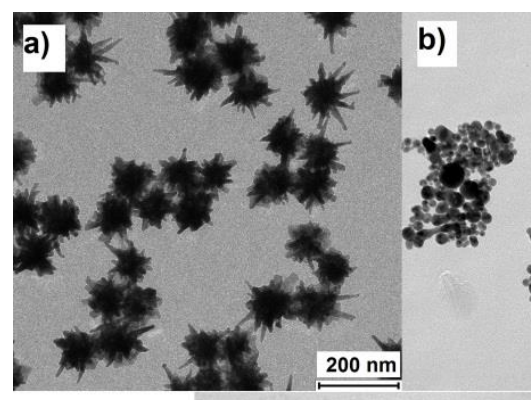

e)

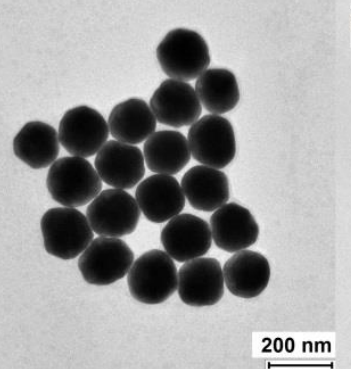

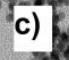

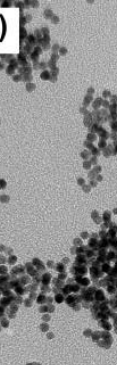

$200 \mathrm{~nm}$

f)

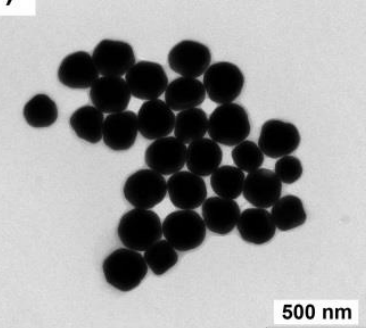

d)

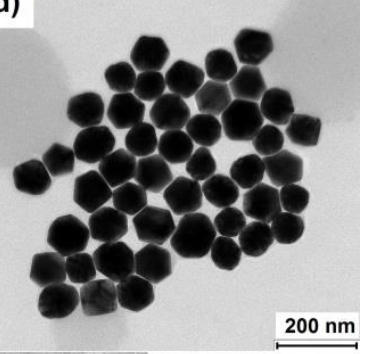

g)
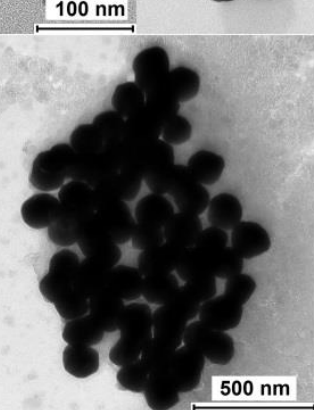

Figure 1 TEM images of the analysed AuNPs: a) Star AuNPs, b) AuSi NCs, c) 10-nm AuNPs in citrate buffer, c) 80-nm AuNPs in citrate buffer, d) 150-nm AuNPs in citrate buffer, e) 200-nm AuNPs in citrate buffer and f) 150-nm AuNPs in PBS buffer.

The size distribution histograms of the analysed particles are presented in Figure 2 a)-g). The star AuNPs showed an average diameter of $79 \mathrm{~nm}$ with spikes excluded. The AuSi NCs were about $16 \mathrm{~nm}$. The size of the 10-nm and 80-nm AuNPs correlated with that announced by the producer. The average diameter of the larger AuNPs (150- and 200-nm) in citrate buffer and 150-nm AuNPs in PBS buffer was approximately 10\% smaller than the average values announced by the producer $(133,179$ and $136 \mathrm{~nm}$, respectively).

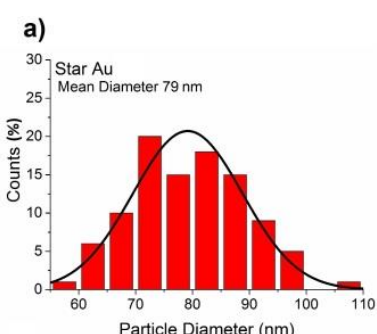

e)

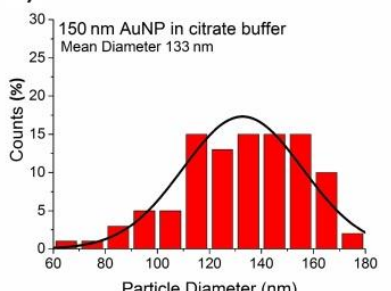

b)

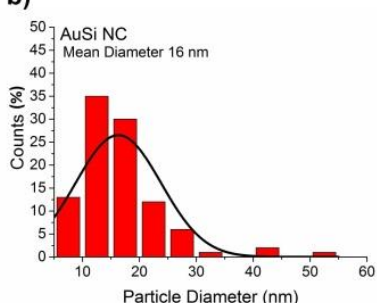

f)
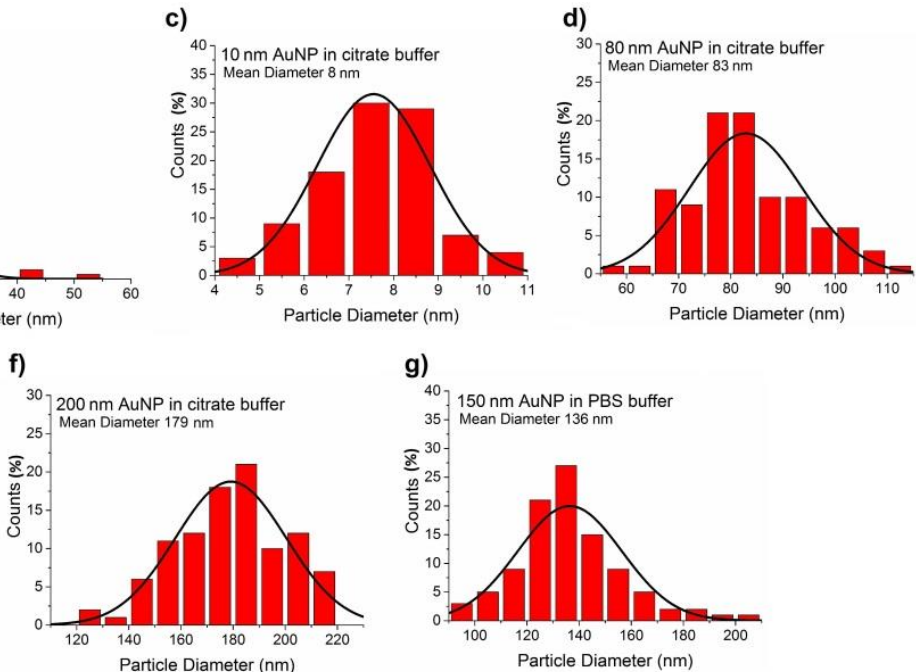
Figure 2 Size histogram for the analysed AuNPs calculated from the TEM images with normal distribution curves: a) Star AuNPs, b) AuSi NCs, c) 10-nm AuNPs, c) 80-nm AuNP, d) 150-nm AuNPs, e) 200-nm AuNPs in citrate buffer and f) 150-nm AuNPs in PBS buffer. 100 particles of each type from 2-5 TEM images were used for calculations.

Since OD of the NPS can indicate the best excitation wavelength band for SERS, through the connection to resonance wavelength of the particles, VIS-NIR spectra for the chosen NPs were recorded (Figure 3). According to the VIS-NIR spectra, star AuNPs and 200-nm AuNPs in citrate buffer show the highest absorbance at 785-nm wavelength, while 10-nm and 80-nm spherical NPs and AuSi NCs - the lowest. Thus, we assumed that the 200-nm particles and star AuNPs would show highest SERS enhancement factors.

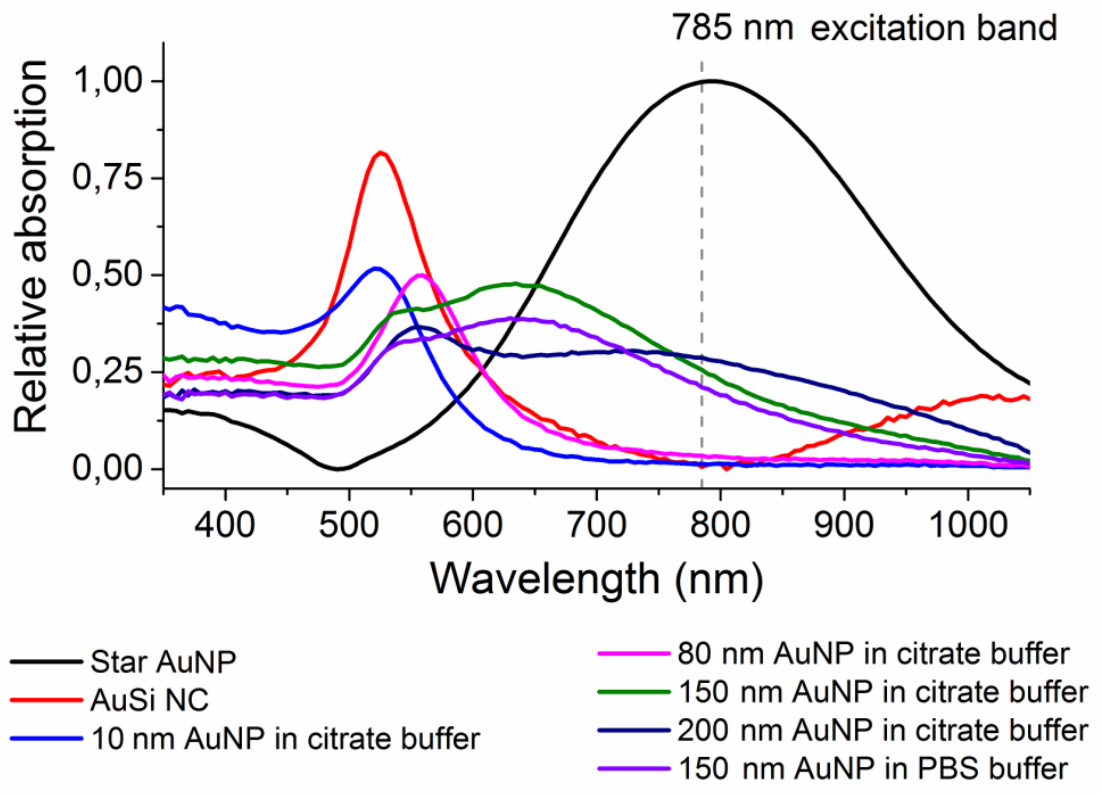

Figure 3 VIS-NIR absorbance spectrum (optical density) for the analysed AuNPs. 
The relative SERS enhancement ability of the AuNPs was further studied by recording the SERS spectra for $\begin{array}{lllll}\text { Raman } & \text { active } & \text { R6G. } & \text { As } & \text { follows }\end{array}$

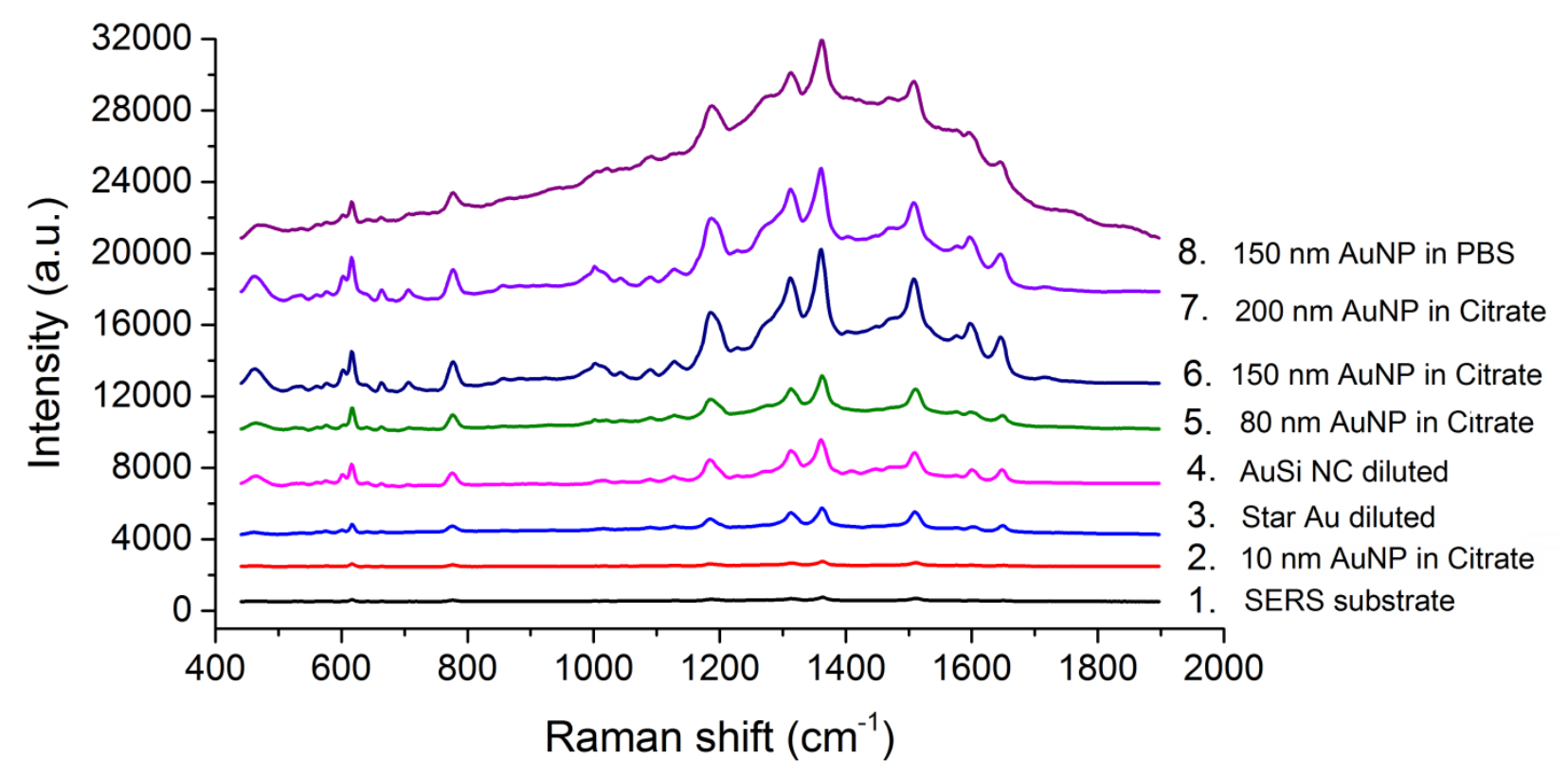

Figure 4, the large spherical AuNPs exhibit highest R6G peaks. The absolutely highest spectrum was achieved with 150-nm AuNPs in PBS, but the peaks have a large fluorescence background originating from the PBS buffer. Such a background can pose a problem handling biological samples and mask Raman peaks of less intensive Raman signals than the used R6G sample. Interestingly, the star AuNPs showing the best absorbance at 785-nm wavelength, show lower enhancement. The signal intensity of R6G with star AuNPs is in the same range as achieved with similar size 80-nm AuNPs. Thus, the spiked shaped of the stars do not show an advantage compared with small molecule detection possibly due to presence of ligands on their surface. Another interesting observation is the peak intensity of R6G with bare AuSi NCs. Although 5 times smaller in diameter, the AuSi NCs have similar enhancement ability as the $80-\mathrm{nm}$ particles, which could be due to the absence of PVP on the surface of the AuSi NCs. This ensures an excellent contact of R6G molecules with metal supressing fluorescence and providing maximal enhancement. Compared to the spherical 10-nm AuNPs with similar diameter range as the AuSi NCs, only 8-nm smaller, AuSi NCs showed ten times higher peak intensity. The R6G signal intensity of 10-nm particles was similar to the signal of SERS substrate showing no additional enhancement. 
From the results of the R6G detection we can conclude that the citrate buffer might be preferential to the PBS buffer as a NP medium as it shows lower fluorescent background signal. The largest particles, 150nm and 200-nm AuNPs, showed the best peak intensity. 80-nm particles, star AuNPs and the AuSi NCs showed lower enhancement, but had 10 times higher signal level than the SERS substrate alone.

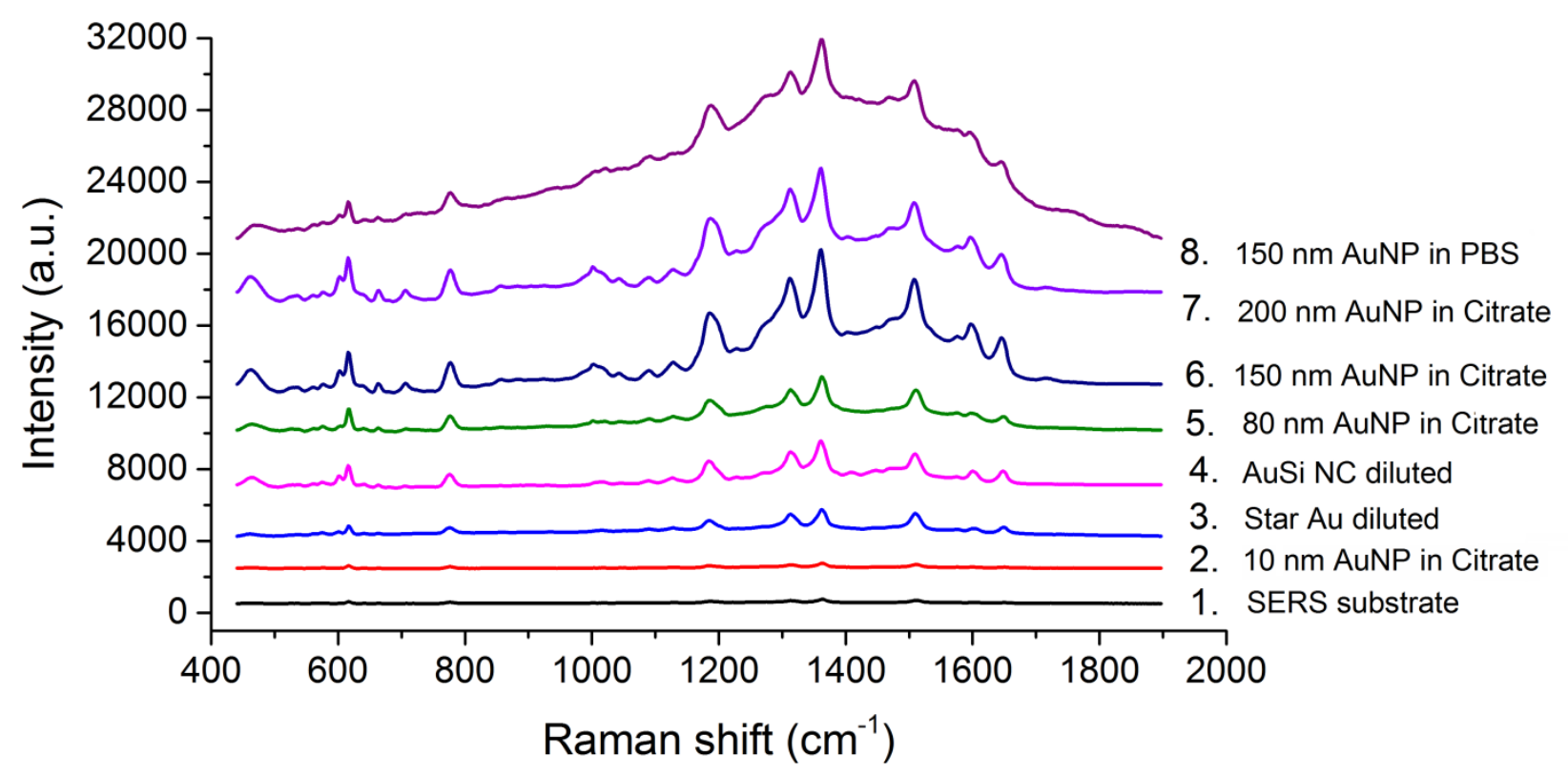

Figure 4 SERS spectra of R6G detected with different AuNPs on top of SERS substrate. The average SERS spectra were calculated from 30 points measured in duplicate. The curves are separated for clarity with arbitrary constant values.

As pointed out in literature, the power of the excitation laser can damage biological cells during the measurement procedure ${ }^{13,40}$. Appropriate laser power also depends on whether the cells are measured in solution or as dried on top of the SERS substrate. As Syamala et al. ${ }^{12}$ reported that the detection of yeast cells incubated with silver colloids had issues in repeatability, the aim of the present study was detection of the cells in thermally dried state with NIR excitation wavelength and low excitation power causing less thermal damage to the cells. Optimal power for the dried yeast cells was studied by recording the SERS spectrum for W. anomalus as a function of laser power. The change in the average intensity of the main Raman peak 738 
$\mathrm{cm}^{-1}$ relative to the used power is presented in Figure $5 \mathrm{a}$ ). Excitation power of $2 \mathrm{~mW}$ requires over $60 \mathrm{~s}$ integration times to show several distinctive peaks for the yeast cells. On the other hand, Figure $5 \mathrm{c}$ ) $-\mathrm{d}$ ) show that laser power higher than 20-mW destroys the characteristic Raman peaks $\left(738 \mathrm{~cm}^{-1}\right.$ and $\left.967 \mathrm{~cm}^{-1}\right)$ seen in Figure $5 \mathrm{~b}$ ). The main Raman peak is not visible anymore with $34 \mathrm{~mW}$ power. Clearly too high laser power can damage the cells, although it does not destroy the buffer-originated fluorescence signal. With $12 \mathrm{~mW}$ power the $738 \mathrm{~cm}^{-1}$ Raman peak has the highest intensity after background reduction and the integration time could be shortened by half from $2 \mathrm{~mW}$ excitation. Thus the best results were achieved with $12 \mathrm{~mW}$ excitation laser power.

a)

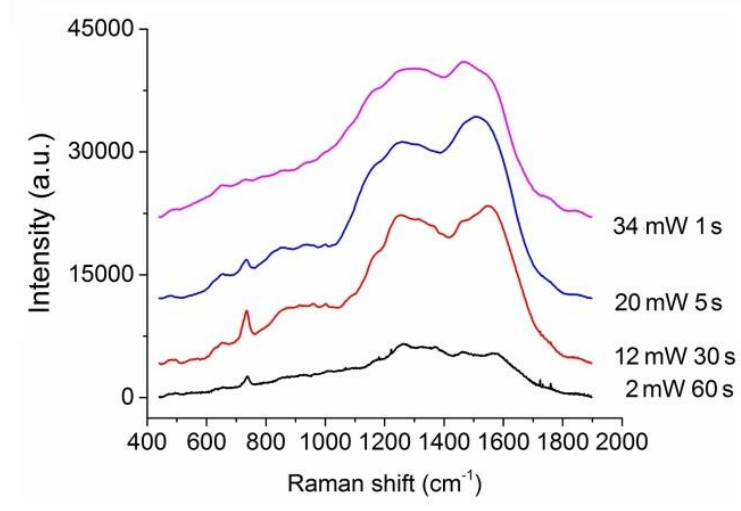

b)

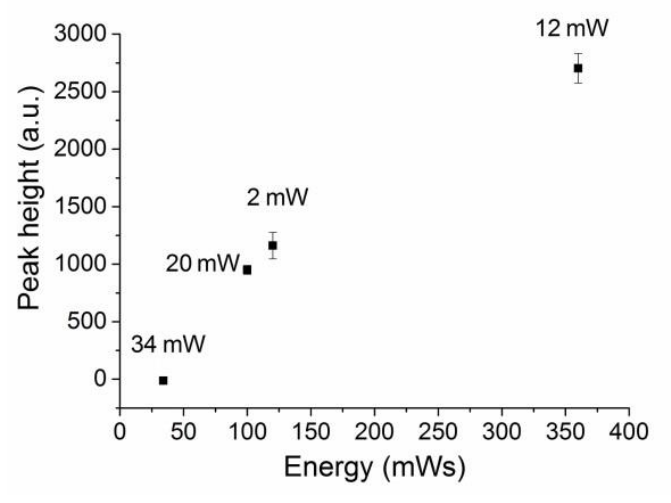

Figure 5 a) SERS spectra of $W$. anomalus detected using 4 laser powers and varying integration times. b) The baseline corrected peak height for the Raman peak $738 \mathrm{~cm}^{-1}$ as a function of energy calculated from the laser power and used integration time. The average SERS spectra were detected using 150-nm AUNPs in citrate buffer on top of SERS substrate and calculated from 15 points measured for each laser power.

The chosen AuNPs were applied to measure the spectrum for $W$. anomalus using a slightly lower (10 $\mathrm{mW}$ ) excitation laser power and reduced total integration time $15 \mathrm{~s}$ to ensure minimal thermal damage. This study included triplicate samples with 10 measurement points per sample to see the repeatability of the detection method. In the graphs in Figure 6 the average deviation between the measurement points is shown with the red curves. As can be seen especially for the 150-nm AuNPs in citrate buffer and the AuSi NCs, the position of the Raman bands remains constant for all measurement points. The SERS substrates alone without AuNPs do not show characteristic peaks such as the $738 \mathrm{~cm}^{-1}$ for $W$. anomalus and the signal intensity 
for the whole spectrum remains low. The star AuNPs and 10-nm spherical AuNPs show similarly low signal intensity without characteristic peaks. The star AuNPs showed a clear signal with the R6G sample (

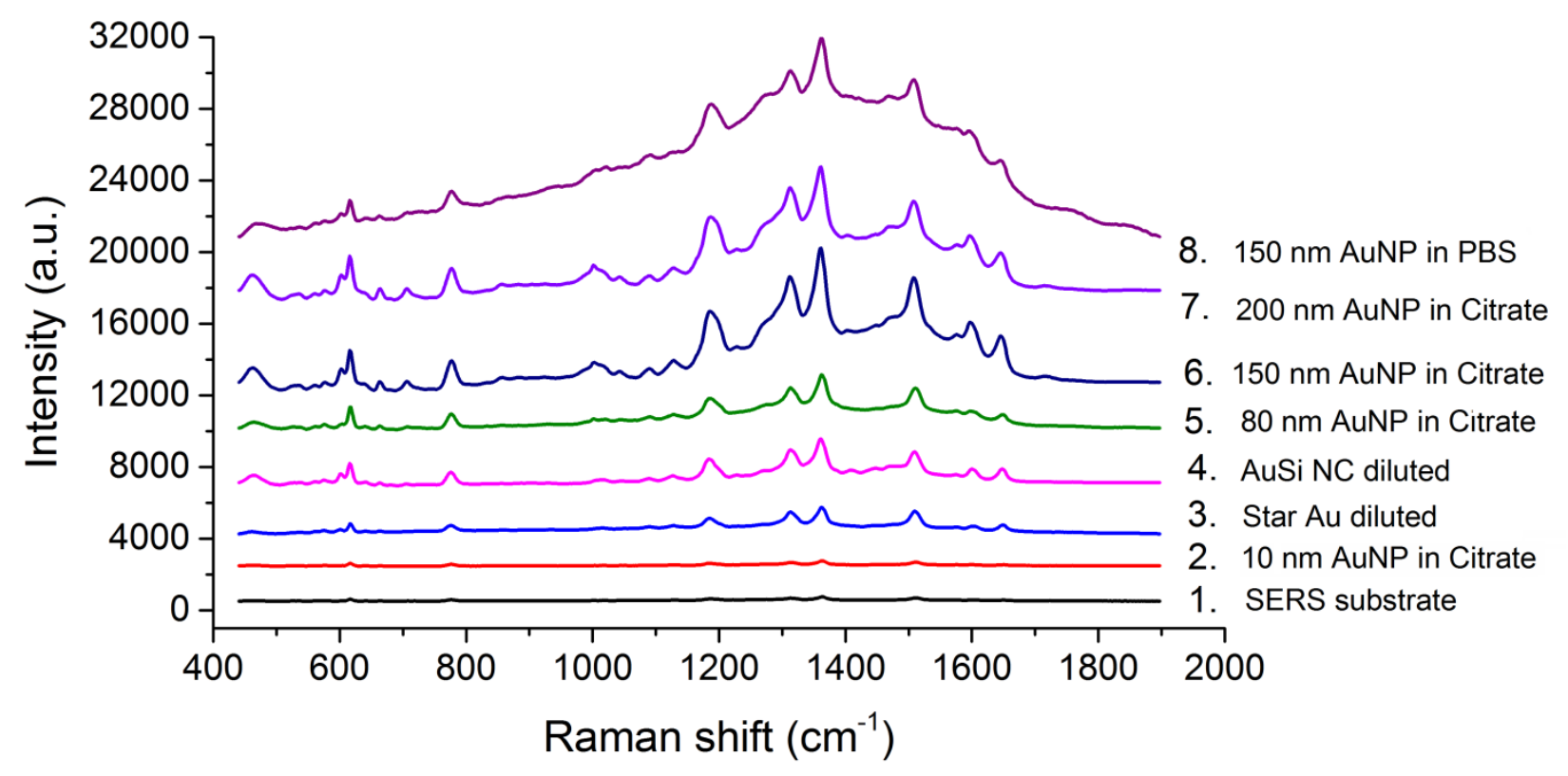

Figure 4) which leads into speculation whether the coverage of the star AuNPs with PVP prevents the contact of the gold surface with the bacteria suppressing the Raman signal. The larger spherical AuNPs in citrate buffer show the characteristic peak $738 \mathrm{~cm}^{-1}$, although for the 150-nm AuNPs the spectrum is more distinct than $80-\mathrm{nm}$ or $200-\mathrm{nm}$ particles. The good suitability, indicated for $200-\mathrm{nm}$ AuNPs by the VIS-NIR absorbance spectrum (Figure 3) does not exhibit higher Raman signals with the actual yeast samples. Additionally, the difference in AuNP buffer can significantly affect the results of the yeast detection, as the $150 \mathrm{~nm}$ AuNPs in PBS do not show any characteristic yeast peaks in contrast to the result with 150nm AuNPs in citrate buffer. This could be due to the Raman signal and the fluorescence background of the PBS buffer. After washing AuNPs and replacing the buffer with DI water (Raman neutral medium), background signal diminishes and the main Raman peak is detected, but the intensity is lower than acquired with similar AuNPs in citrate buffer. The results obtained with the ultra-pure composite AuSi NCs gave the lowest background signal for the $W$. anomalus and in this respect are optimal for the yeast detection. 


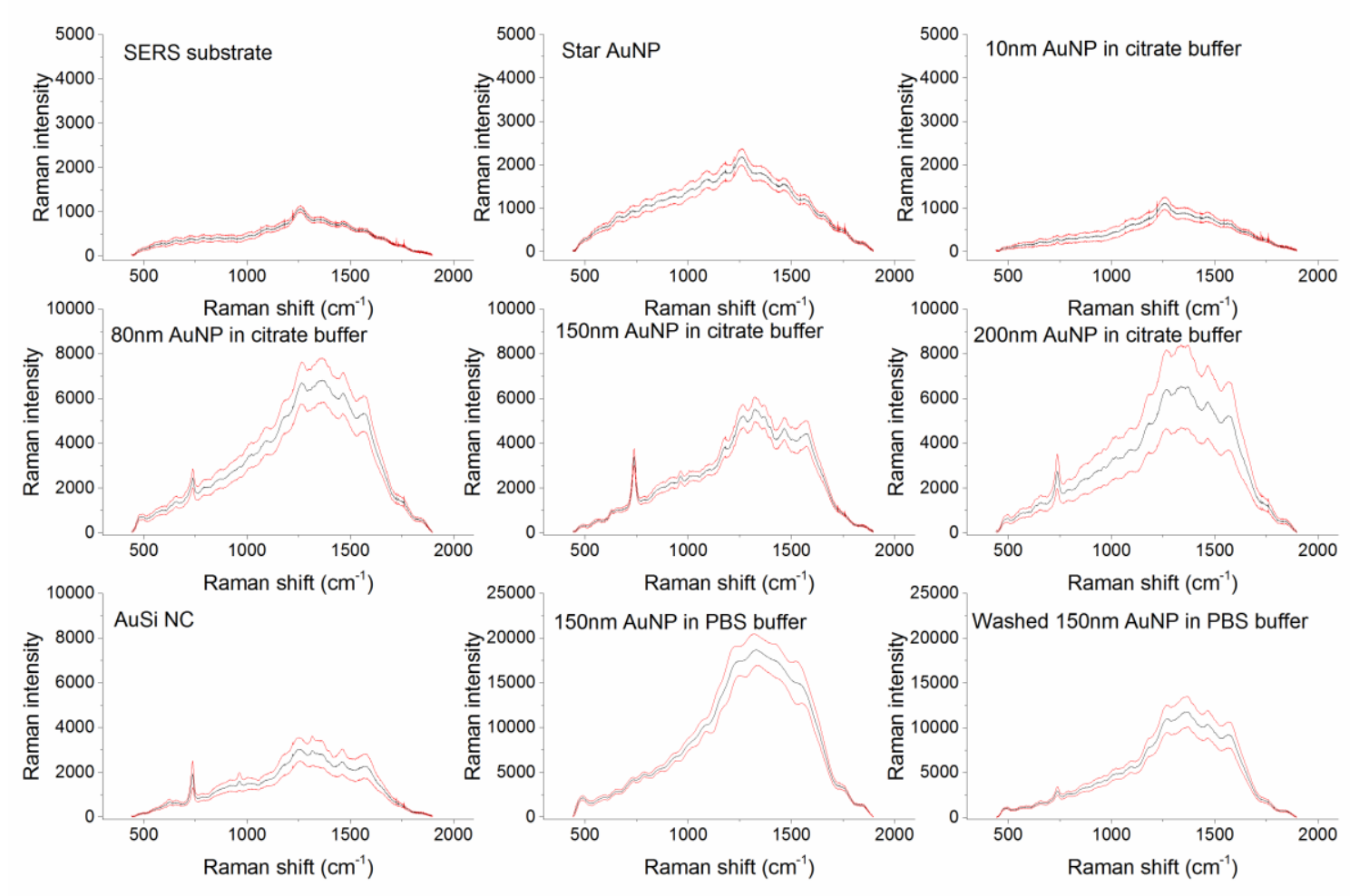

Figure 6 SERS spectra of $\boldsymbol{W}$. anomalus with average absolute deviations detected using different AuNPs on top of SERS substrate. The average SERS spectra were calculated from 10 points measured in triplicate.

The AuSi NCs, with the original concentration, were used to detect the SERS spectrum for W. anomalus, $B$. bruxellensis and R. mucilaginosa. The background SERS spectrum for the physiological saline was measured and subtracted from the spectra of the yeasts. The samples were dried on top of SERS substrate with subsequent adding of AuSi NCs. The recorded yeast SERS spectra are shown in Figure 7, where several Raman bands for the yeast strains are clearly distinguishable. The pink-pigmened R. mucilaginosa showed 7 characteristic peaks, while the spoilage yeasts $W$. anomalus and B. bruxellensis showed 4 peaks. This could be related to the higher absorption of excitation light due to $R$. mucilaginosa color pigment. 

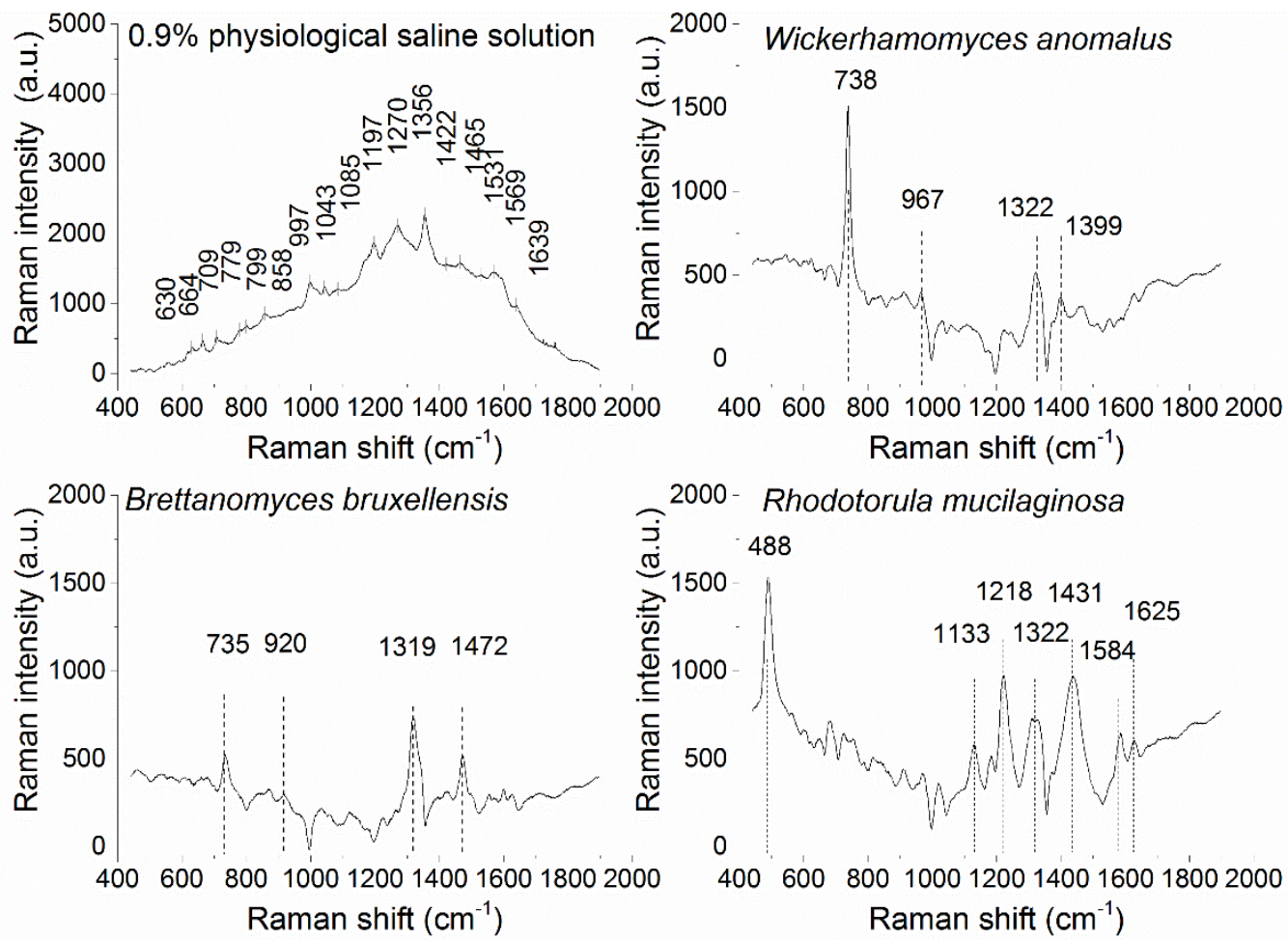

Figure 7 a) SERS spectra of $0.9 \%$ physiological saline. SERS spectra of b) W. anomalus, c) B. bruxellensis and d) $R$.

mucilaginosa detected on top of SERS substrate with AuSi NCs. The average SERS spectra were recorded in triplicate using total $15 \mathrm{~s}$ integration time. Origin Pro user defined baseline reduction was utilised to remove fluorescence background (shown for $W$. anomalus in Figure 6).

The detected Raman bands with preliminary band assignments are presented in Table 1. Typically the yeast cell wall is composed of $25 \%$ helical b(1-3) and b(1-6)-D-glucans, $25 \%$ oligomannans, $20 \%$ protein, $10 \%$ lipids, and some chitin. The proteins present are predominantly mannoprotein complexes $^{8}$. To our knowledge, this is a first study showing the Raman spectra for W. anomalus. Four characteristics peaks were detected at $738 \mathrm{~cm}^{-1}, 967 \mathrm{~cm}^{-1}, 1322 \mathrm{~cm}^{-1}$ and $1399 \mathrm{~cm}^{-1}$. The possible assignments for the detected bands are related to C-S bond and $\mathrm{C}-\mathrm{N}$ stretch, and to protein and lipid bands. B. bruxellensis showed 4 characteristics peaks situated at $735 \mathrm{~cm}^{-1}, 920 \mathrm{~cm}^{-1}, 1319 \mathrm{~cm}^{-1}$ and $1472 \mathrm{~cm}^{-1}$. Although the peaks for the spoilage yeasts were in similar regions they were slightly shifted allowing an easy discrimination. When comparing the B. bruxellensis result to the study conducted previously by S.B. Rodriguez et $a l .{ }^{5}$, the detected spectrum has more distinctive Raman lines. This could be related to the use of NIR excitation and lower excitation laser power as the cells do not need to endure strong thermal damage. This shows how the use of 
thermally dried cells can be beneficial in detecting cell wall features. The B. bruxellensis peaks seemed to originate from sugars, proteins and lipids. The $R$. mucilaginosa Raman spectrum has been studied previously with Raman microimaging ${ }^{40,41}$ and by SERS with active silver coated glass fiber tips. ${ }^{13}$ The detection method here revealed several Raman bands for $R$. mucilaginosa originating from polysaccharides, lipids and hemoproteins. The features of the cell, responsible for the detected peaks, are listed next to the band assignments. ${ }^{41}$ In contrary to the studies conducted by R. Gessner et al. the characteristic peaks for the carotenoids ${ }^{13}$ at Raman shifts $1001 \mathrm{~cm}^{-1}, 1151 \mathrm{~cm}^{-1}$ and $1508 \mathrm{~cm}^{-1}$ are missing. The absence of these peaks is most probably attributed to the fact that the excitation light $(785 \mathrm{~nm})$ does not match the carotenoid absorption spectrum. Although this wavelength can be preferable with non-pigmented yeasts due to reduced fluorescence, the carotenoid Raman peaks are suppressed with NIR wavelengths as indicated by P. Rösch et $a^{40}{ }^{40}$ in the case of Micrococcus luteus. However, the spectrum detected here showed several characteristic peaks for the R. mucilaginosa which can be used for yeast discrimination.

Table 1 Raman band assignments ${ }^{8}$ for the spectra of $W$. anomalus, B. bruxellensis and $R$. mucilaginosa and reference biomolecule listing for $R$. mucilaginosa.

\begin{tabular}{|c|c|c|c|c|}
\hline $\begin{array}{l}\text { Raman } \\
\text { band }\end{array}$ & W. anomalus & B. bruxellensis & R. mucilaginosa & $\begin{array}{l}\text { Study by M. Z. Pacia } \\
\text { et al. } 41\end{array}$ \\
\hline 488 & ---------------------------------- & ---------------------------------- & Polysaccharides & $\begin{array}{l}\text { Outside detection } \\
\text { range }\end{array}$ \\
\hline $735 / 738$ & $\begin{array}{l}\text { Adenine from flavin, C- } \\
\mathrm{S}, \mathrm{N} \text {-acetyl-D- } \\
\text { glucosamine }\end{array}$ & $\begin{array}{l}\text { Adenine from flavin, } \mathrm{C}- \\
\mathrm{S}, \mathrm{N} \text {-acetyl-D- } \\
\text { glucosamine }\end{array}$ & 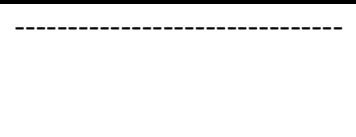 & \\
\hline 920 & ------------------------------- & Glucose & ------------------------------- & \\
\hline 967 & C-N stretch & |------------------------------ & |------------------------------- & \\
\hline 1133 & --------------------------------- & ------------------------------- & Lipid & Hemoprotein, cell wall \\
\hline 1218 & |------------------------------ & |-------------------------------- & Amide III & Hemoprotein, cell wall \\
\hline $1319 / 1322$ & $\mathrm{C}-\mathrm{H}$ bend protein & $\mathrm{C}-\mathrm{H}$ bend protein & $\mathrm{C}-\mathrm{H}$ bend protein & $\begin{array}{l}\text { Lipid or hemoprotein, } \\
\text { cell wall }\end{array}$ \\
\hline 1399 & $\begin{array}{l}\mathrm{CH}_{2} \text { bend (protein, } \\
\text { lipid) }\end{array}$ & 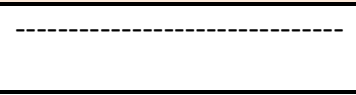 & 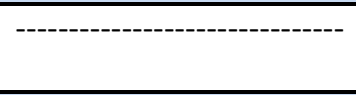 & \\
\hline 1431 & --------------------------------- & ---------------------------------- & $\begin{array}{l}\mathrm{CH}_{2} \text { bend (protein, } \\
\text { lipid) }\end{array}$ & Lipid, cell wall \\
\hline 1472 & 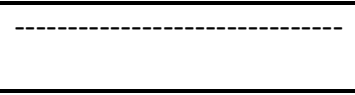 & $\begin{array}{l}\mathrm{CH}_{2} \text { bend (protein, } \\
\text { lipid) }\end{array}$ & --------------------------------- & \\
\hline 1584 & 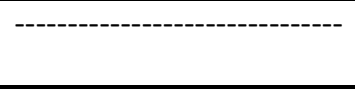 & 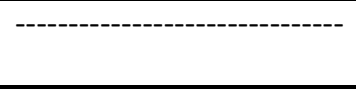 & $\begin{array}{l}\mathrm{N}-\mathrm{H}, \mathrm{C}-\mathrm{H} \text { bend, } \mathrm{C}=\mathrm{C} \\
\text { stretch }\end{array}$ & Lipid, cell wall \\
\hline 1625 & ----------------------------- & ----------------------------- & Tyrosine, tryptophan & Hemoprotein, cell wall \\
\hline
\end{tabular}


The developed SERS detection method makes the discrimination of the yeast cells of $W$. anomalus, $B$. bruxellensis and R. mucilaginosa very straightforward as the detected Raman peaks of different yeasts are clearly shifted from each other. Thus, there is no need for chemometric calculations such as multivariate principal components analysis (PCA $)^{42}$ or unsupervised hierarchical cluster analysis $(\mathrm{HCA})^{43}$ to separate the samples using peak intensities of similar spectrums to identify the yeasts from each other. In this study, yeast were cultivated on plate in rich culture medium and harvested at relatively high concentration $\left(10^{7} \mathrm{cells} / \mathrm{ml}\right)$ into physiological saline. The method can be implemented for detection of lower cell counts and discrimination of yeasts at strain level utilising improved data analysis and additional cell accumulation methods such as filtration. ${ }^{44}$

\section{Conclusions}

We recorded characteristic Raman spectra for yeast strains of $W$. anomalus, B. bruxellensis and $R$. mucilaginosa using gold nanoparticles on top of SERS substrate. Our study revealed that thermally dried yeast cells of different species could be clearly discriminated with near-infrared excitation without a need for chemometric calculations. The analysis of different AuNPs showed that the effect of the buffer of the gold nanoparticle suspensions to the acquired spectra could overflow the spectra and hide the Raman lines of the yeasts. Care has to be taken to avoid incompatible buffers with the nanoparticles and yeast solutions when analysing yeast strains with Raman spectroscopy. Additionally, PVP cover of the gold star-like nanoparticles substantially decreased particle Raman enhancement efficacy. Large spherical gold nanoparticles (150-nm) in citrate buffer and the ultrapure composite nanoparticles showed promising SERS enhancement capability for yeast detection. Bare AuSi composite nanoparticles were used for detection of $W$. anomalus, $B$. bruxellensis and R. mucilaginosa and distinctive spectra were recorded for all yeast species. The developed SERS detection method provides a simple and straightforward yeast discrimination tool, in particular, for food and beverage industry. 


\section{Acknowledgments}

This project was funded by VTT government grant through SENSFOOD-project and by Academy of Finland through M-SPEC project (284907) and partially by projects 260321 and 290596 (Academy of Finland). The authors thank the support from Infotech Oulu Graduate School. O. Bibikova acknowledges the support from International Graduate School in Molecular Medicine Ulm. This work was partially supported by Government of Russian Federation (Grant 074-U01). Yu. Ryabchikov and A. V. Kabashin acknowledge a support from LASERNANOCANCER (No. PC201420) and GRAVITY projects of the ITMO “Plan Cancer 2014-2019” of INSERM and of the AMIDEX project (No ANR-11-IDEX-0001-02) funded by the French Government program.

\section{References}

1. Loureiro, V. Spoilage yeasts in foods and beverages: characterisation and ecology for improved diagnosis and control. Food Res. Int. 33, 247-256 (2000).

2. Loureiro, V. \& Querol, A. The prevalence and control of spoilage yeasts in foods and beverages. Trends Food Sci. Technol. 10, 356-365 (1999).

3. Stratford, M. Food and beverage spoilage yeasts. Yeasts in food and beverages (Springer Berlin Heidelberg, 2006). at <http://link.springer.com/content/pdf/10.1007/978-3-540-28398-0_11.pdf>

4. Amorim, M. et al. Nutritional ingredients from spent brewer's yeast obtained by hydrolysis and selective membrane filtration integrated in a pilot process. J. Food Eng. 185, 42-47 (2016).

5. Rodriguez, S. B., Thornton, M. A. \& Thornton, R. J. Raman Spectroscopy and Chemometrics for Identification and Strain Discrimination of the Wine Spoilage Yeasts Saccharomyces cerevisiae, Zygosaccharomyces bailii, and Brettanomyces bruxellensis. Appl. Environ. Microbiol. 79, 6264-6270 (2013).

6. Pitt, J. I. \& Hocking, A. D. in Fungi and Food Spoilage 19-52 (Springer US, 2009). doi:10.1007/978-0387-92207-2_4

7. Loureiro, V. \& Malfeito-Ferreira, M. Spoilage yeasts in the wine industry. Int. J. Food Microbiol. 86, 23- 
50 (2003).

8. Sayin, I., Kahraman, M., Sahin, F., Yurdakul, D. \& Culha, M. Characterization of Yeast Species Using Surface-Enhanced Raman Scattering. Appl. Spectrosc. Vol. 63, Issue 11, pp. 1276-1282 63, $1276-$ $1282(2009)$.

9. Chrimes, A. F. et al. In situ SERS probing of nano-silver coated individual yeast cells. Biosens. Bioelectron. 49, 536-541 (2013).

10. Sujith, A. et al. Surface enhanced Raman scattering analyses of individual silver nanoaggregates on living single yeast cell wall. Appl. Phys. Lett. 92, 103901 (2008).

11. Chen, J. et al. Fabrication of Large-Area, High-Enhancement SERS Substrates with Tunable Interparticle Spacing and Application in Identifying Microorganisms at the Single Cell Level. (2012).

12. Syamala, K. M. et al. Inhibition Assay of Yeast Cell Walls by Plasmon Resonance Rayleigh Scattering and Surface-Enhanced Raman Scattering Imaging. (2012).

13. Gessner, R., Rösch, P., Kiefer, W. \& Popp, J. Raman spectroscopy investigation of biological materials by use of etched and silver coated glass fiber tips. Biopolymers 67, 327-330 (2002).

14. Anker, J. N. et al. Biosensing with plasmonic nanosensors. Nat. Mater. 7, 442-453 (2008).

15. Culha, M., Kahraman, M., Çam, D., Sayın, I. \& Keseroğlu, K. Rapid identification of bacteria and yeast using surface-enhanced Raman scattering. Surf. Interface Anal. 42, 462-465 (2010).

16. Sujith, A. et al. Imaging the cell wall of living single yeast cells using surface-enhanced Raman spectroscopy. Anal. Bioanal. Chem. 394, 1803-1809 (2009).

17. Adam M. Schwartzberg et al. Unique Gold Nanoparticle Aggregates as a Highly Active SurfaceEnhanced Raman Scattering Substrate. (2004).

18. Greulich, C. et al. The toxic effect of silver ions and silver nanoparticles towards bacteria and human cells occurs in the same concentration range. RSC Adv. 2, 6981 (2012).

19. Alkilany, A. M. \& Murphy, C. J. Toxicity and cellular uptake of gold nanoparticles: what we have learned 
so far? J. Nanoparticle Res. 12, 2313-2333 (2010).

20. Ravi Shukla et al. Biocompatibility of Gold Nanoparticles and Their Endocytotic Fate Inside the Cellular Compartment: A Microscopic Overview. (2005).

21. Notingher, I. et al. In situ Characterisation of Living Cells by Raman Spectroscopy. Spectroscopy 16, 43-51 (2002).

22. Li, M. et al. Three-Dimensional Hierarchical Plasmonic Nano-Architecture Enhanced SurfaceEnhanced Raman Scattering Immunosensor for Cancer Biomarker Detection in Blood Plasma. (2013).

23. Uusitalo, S. et al. Detection of Listeria innocua on roll-to-roll produced SERS substrates with gold nanoparticles. RSC Adv. 6, 62981-62989 (2016).

24. Oo, S. Z. et al. Disposable plasmonic plastic SERS sensor. Opt. Express 21, 18484-91 (2013).

25. Yuan, H. et al. Gold nanostars: surfactant-free synthesis, 3D modelling, and two-photon photoluminescence imaging. Nanotechnology 23, 75102 (2012).

26. Bibikova, O. et al. Plasmon-Resonant Gold Nanostars With Variable Size as Contrast Agents for Imaging Applications. IEEE J. Sel. Top. Quantum Electron. 22, 13-20 (2016).

27. Bibikova, O. et al. Optical properties of plasmon-resonant bare and silica-coated nanostars used for cell imaging. J. Biomed. Opt. 20, 076017 (2015).

28. Blandin, P. et al. Femtosecond laser fragmentation from water-dispersed microcolloids: toward fast controllable growth of ultrapure Si-based nanomaterials for biological applications. J. Mater. Chem. B 1, 2489 (2013).

29. Rioux, D. et al. Silicon nanoparticles produced by femtosecond laser ablation in water as novel contamination-free photosensitizers. J. Biomed. Opt. 14, 21010 (2009).

30. Maximova, K. et al. Size-controllable synthesis of bare gold nanoparticles by femtosecond laser fragmentation in water. Nanotechnology 26, 65601 (2015).

31. Kabashin, A. V. \& Meunier, M. Synthesis of colloidal nanoparticles during femtosecond laser ablation 
of gold in water. J. Appl. Phys. (2003).

32. Ryabchikov, Y. V., Popov, A. A., Sentis, M., Timoshenko, V. Y. \& Kabashin, A. V. Structural properties of gold-silicon nanohybrids formed by femtosecond laser ablation in water at different fluences. in (eds. Kabashin, A. V., Geohegan, D. B. \& Dubowski, J. J.) 97370F (International Society for Optics and Photonics, 2016). doi:10.1117/12.2217777

33. Hebié, S. et al. Advanced Electrocatalysts on the Basis of Bare Au Nanomaterials for Biofuel Cell Applications. (2015).

34. Angelov, S. D. et al. Electrophoretic deposition of ligand-free platinum nanoparticles on neural electrodes affects their impedance in vitro and in vivo with no negative effect on reactive gliosis. $J$. Nanobiotechnology 14, 3 (2016).

35. Martin, E. et al. Isolation of a Wickerhamomyces anomalus yeast strain from the sandfly Phlebotomus perniciosus, displaying the killer phenotype. Med. Vet. Entomol. 30, 101-106 (2016).

36. Hayashi, N., Arai, R., Tada, S., Taguchi, H. \& Ogawa, Y. Detection and identification of Brettanomyces/Dekkera sp. yeasts with a loop-mediated isothermal amplification method. Food Microbiol. 24, 778-785 (2007).

37. Aksu, Z. \& Eren, A. T. Carotenoids production by the yeast Rhodotorula mucilaginosa: Use of agricultural wastes as a carbon source. Process Biochem. 40, 2985-2991 (2005).

38. Laitila, A. et al. Yeasts in malting, with special emphasis on Wickerhamomyces anomalus (synonym Pichia anomala). Antonie Van Leeuwenhoek 99, 75-84 (2011).

39. Hering, K. et al. SERS: a versatile tool in chemical and biochemical diagnostics. Anal. Bioanal. Chem. 390, 113-124 (2008).

40. Rösch, P., Schmitt, M., Kiefer, W. \& Popp, J. The identification of microorganisms by micro-Raman spectroscopy. J. Mol. Struct. 661, 363-369 (2003).

41. Pacia, M. Z. et al. Interplay between carotenoids, hemoproteins and the 'life band' origin studied in live Rhodotorula mucilaginosa cells by means of Raman microimaging. Analyst 140, 1809-1813 (2015). 
42. Guicheteau, J., Christesen, S., Emge, D. \& Tripathi, A. Bacterial mixture identification using Raman and surface-enhanced Raman chemical imaging. J. Raman Spectrosc. 41, 1632-1637 (2010).

43. Meisel, S., Stöckel, S., Rösch, P. \& Popp, J. Identification of meat-associated pathogens via Raman $\begin{array}{llll}\text { microspectroscopy. } & \text { Food } & \text { (2014). at }\end{array}$ <http://www.sciencedirect.com/science/article/pii/S0740002013001615>

44. Lemma, S. M. et al. Removal of bacteria and yeast in water and beer by nylon nanofibrous membranes. J. Food Eng. 157, 1-6 (2015). 